\title{
RNA-seq analysis reveals alternative splicing under salt stress in cotton, Gossypium davidsonii
}

\author{
Guozhong Zhu, Weixi Li, Feng Zhang and Wangzhen Guo*id
}

\begin{abstract}
Background: Numerous studies have focused on the regulation of gene expression in response to salt stress at the transcriptional level; however, little is known about this process at the post-transcriptional level.

Results: Using a diploid D genome wild salinity-tolerant cotton species, Gossypium davidsonii, we analyzed alternative splicing (AS) of genes related to salt stress by comparing high-throughput transcriptomes from salt-treated and wellwatered roots and leaves. A total of 14,172 AS events were identified involving 6798 genes, of which intron retention (35.73\%) was the most frequent, being detected in 3492 genes. Under salt stress, 1287 and 1228 differential alternative splicing (DAS) events were identified in roots and leaves, respectively. These DAS genes were associated with specific functional pathways, such as "responses to stress", "metabolic process" and "RNA splicing", implying that AS represents an important pathway of gene regulation in response to salt stress. Several salt response genes, such as pyrroline-5carboxylate synthase (P5CS), $\mathrm{K}^{+}$channel outward (KCO1), plasma membrane intrinsic protein (PIP) and WRKY33 which were involved in osmotic balance, ion homeostasis, water transportation and transcriptional regulation, respectively, were identified with differential alternative splicing under salt stress. Moreover, we revealed that 13 genes encoding Ser/Arg-rich (SR) proteins related to AS regulation were differentially alternatively spliced under salt stress.
\end{abstract}

Conclusion: This study first provide a comprehensive view of AS in G. davidsonii, and highlight novel insights into the potential roles of AS in plant responses to salt stress.

Keywords: RNA-seq, Alternative splicing, Splicing factor, Salt stress, G. davidsonii

\section{Background}

The removal of introns from immature mRNA by a process called "pre-mRNA splicing" occurs in the vast majority of eukaryotic protein-coding genes. In this process, particular exons of a gene may be included in or excluded from the final, processed messenger RNA (mRNA) from that gene. This process is known as alternative splicing (AS) [1]. AS is a ubiquitous mechanism in higher eukaryotes and contributes to both transcriptome and proteome diversity [2]. AS creates multiple mRNA transcripts from a single gene through the selection and utilization of alternative splice sites in the pre-mRNA via different splicing events, including exon skipping (ES), alternative donor site (AD), alternative

\footnotetext{
* Correspondence: moelab@njau.edu.cn

State Key Laboratory of Crop Genetics \& Germplasm Enhancement, Hybrid Cotton R \& D Engineering Research Center, Ministry of Education, Nanjing Agricultural University, Nanjing 210095, China
}

acceptor site (AA), intron retention (IR) and other complicated forms of splicing [3]. The frequency of AS events varies significantly and some are gene- or species-specific. In animals, ES and IR are the most and least frequent, respectively. For example, approximately $35.2 \%$ of all AS events in humans are caused by ES, but only $0.01 \%$ by IR [4]. In contrast, IR is the most predominant form of AS in Arabidopsis [5], Zea mays [6], and Gossypium raimondii [7], whereas ES only accounts for a small proportion.

AS is accomplished by spliceosomes, which are high molecular weight complexes that are assembled at every intron $[8,9]$. They contain five small nuclear ribonucleoprotein particles (snRNPs) and over 200 additional proteins. The identification of splice sites under particular cellular conditions is related to the interaction of additional proteins, globally designated as splicing factors (SFs), that guide spliceosomal components and thereby 
the spliceosome to the respective splice sites [10]. The main families of these SFs are the Ser/Arg-rich (SR) proteins and heterogeneous nuclear ribonucleoprotein particle (hnRNP) proteins. These proteins bind specific sequences in the pre-mRNA called intronic or exonic splicing enhancer or suppressor sequences [11]. Splice site selection reflects the relative occupation of these sequences and interactions between different proteins on a pre-mRNA molecule. In both animals and plants, many SFs/RNA binding proteins (RBPs) and some core spliceosomal components themselves undergo AS in response to signals, and control their own levels and those of other SFs via AS [12, 13]. In addition, the activity of SFs can be regulated by posttranslational modification in response to environmental cues [14].

AS is involved in many physiological processes, as well as responses to biotic and abiotic stresses in plants $[12,15,16]$. Since the significance of AS from SR protein splicing factor was reported [17], many studies had focused on how AS influences important developmental and signaling pathways. It has been demonstrated that ultraviolet (UV) irradiation can induce cell apoptosis by affecting the expression of apoptotic genes in an AS-dependent way [18]. Splicing variants of ABI3 were influenced by a plant splicing factor (SUA) in seed germination, implying that AS participates in both ABA signaling and response to abiotic stresses [19]. Transcriptomic analysis accelerates the identification of new splicing junctions [20]. Using RNA-seq data, genome-wide AS analysis has been conducted in several plant species, such as Oryza sativa [21], Zea mays [6], Glycine max [22] and Arabidopsis [5]. The potential roles of AS in the response to salt stress in Arabidopsis [23] and to heat stress in Physcomitrella patens have been further elucidated [24]. In addition, some splice variants of SR proteins, which are important splicing regulators, were identified in Arabidopsis under high- and low-temperature stress, and they might, in turn, alter the splicing of other pre-mRNAs $[12,25]$. These findings demonstrated that AS is influenced by abiotic stress and, in turn, AS also plays a role in regulating gene expression.

Salinity is one of the most brutal environmental stresses that can hamper crop productivity worldwide. Although cotton is a relatively salt-tolerant species, its growth and development can still be greatly affected by adverse salt conditions [26]. Based on the analysis of the evolution and domestication of cotton using the sequenced G. hirsutum acc. TM-1 genome, it has been implied that the D-subgenome of tetraploid cotton species is a major donor for stress tolerance [7]. Although the mechanism of AS in G. raimondii has been investigated, little is known about AS in response to salt stress in cotton. In the previous study, using transcriptomes comparison from salt-treated and well-watered roots and leaves of G. davidsonii, a diploid D-genome wild salinity-tolerant cotton species, we detected that salt overly sensitive (SOS) and reactive oxygen species (ROS) signaling pathways were involved in salt stress tolerance, and photosynthesis pathways and metabolism played important roles in ion homeostasis and oxidation balance. We also found that alternative splicing is induced by salt stress in cotton [26]. However, deeper analysis between AS and salt stress remains to be investigated. Here, based on the high-throughput transcriptomes from salttreated and well-watered roots and leaves in G. davidsonii, we systematically investigated the global dynamics of AS and elucidated the relationship between AS and salt stress in cotton. We found that the number of AS events under stress conditions was higher than that under normal conditions, indicating that AS plays important roles in the response to salt stress. We also detected SRs involved in AS regulation and investigated their differential expression and AS under salt stress. This study not only provides a comprehensive view of AS in cotton, but also highlights novel insights into the potential roles of AS in plant responses to salt stress.

\section{Results}

\section{Prediction of gene isoforms}

It is well known that multiple mRNA transcripts, also called gene isoforms, can be generated from a single gene via splicing events. Using RNA-seq data (Accessions: SRP061663), which were collected from both roots and leaves at $12,24,48,96$, and $144 \mathrm{~h}$ post salt stress $(200 \mathrm{mM} \mathrm{NaCl})$ in $\mathrm{G}$. davidsonii, with data in normal conditions as controls [26], the splicing events involved in novel exons and novel intergenic transcripts were identified by mapping these data to the sequenced G. raimondii genome. The RNA-seq assays revealed 202,762 isoforms and 47,698 unigenes in total 40 libraries. Nevertheless, expression profiles revealed most isoforms were low abundance, with $70 \%$ lower than one fragments per kilobase per million reads (FPKM), about $20 \%$ between 1 and 10 FPKM, and less than $10 \%$ is over 10 FPKM (Fig. 1a). Expression level below 1 FPKM is thought to be beyond the limit of protein detection [27-29]. It means more than 70 percentage isoforms predicted in our data will not be translated into function proteins. This explains why the number of detected proteins is much less than the number of predicted isoforms. Meanwhile, it also means the low abundance isoforms cannot represent the function of multi-isoforms genes. In order to investigate the proportion of each isoform in corresponding gene, we grouped 40 libraries into four groups, the roots of wellwatered control plants $(\mathrm{RC})$, the roots of salt-stressed plants (RS), the leaves of well-watered controls plants (LC) and the leaves of salt-stressed plants (LS), and performed the statistics of top five most abundant isoforms for multi- 


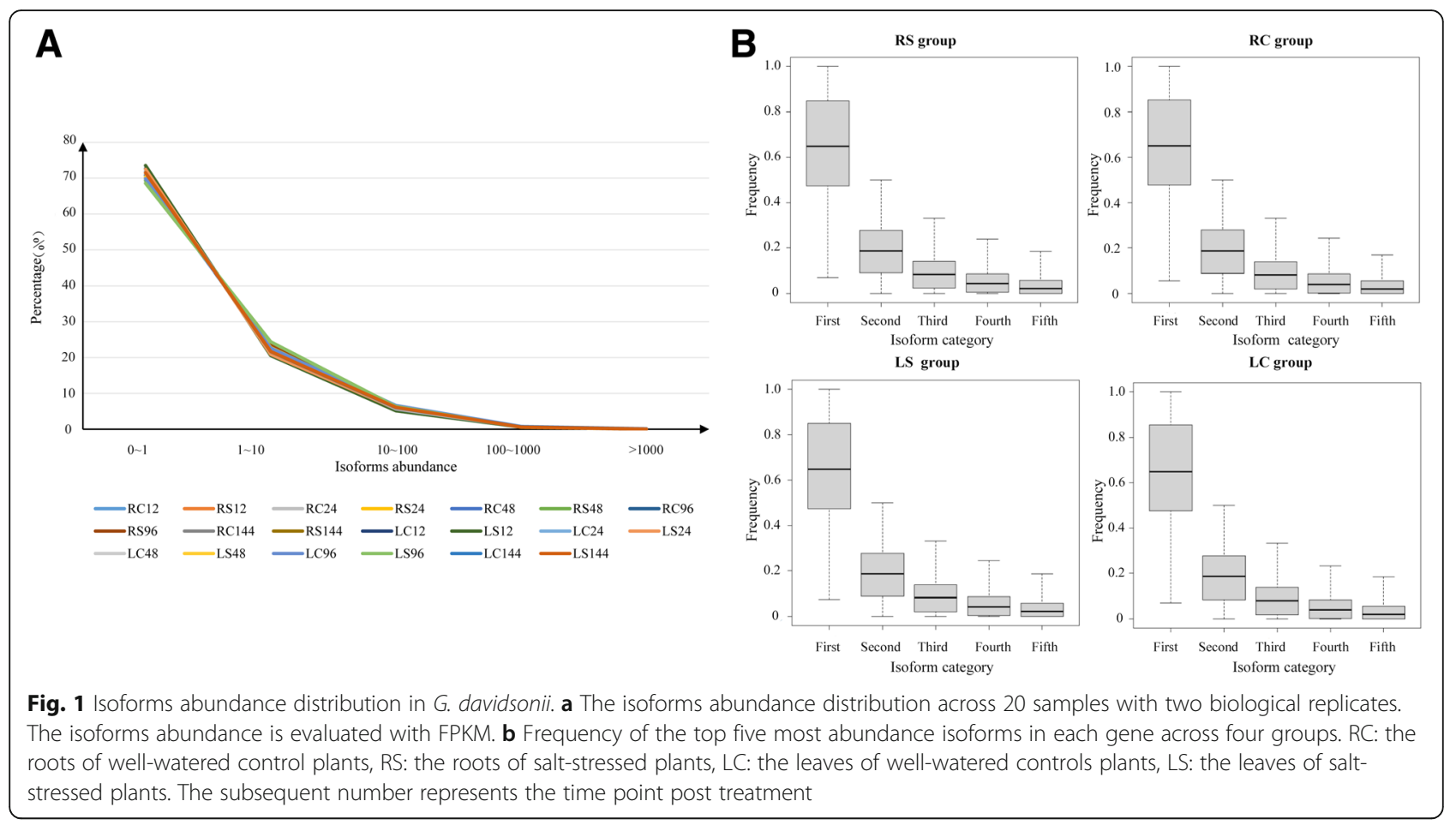

isoforms genes (Fig. 1b and Additional file 1: Figure S1). Top1 isoforms in each group occupied greater $60 \%$ gene abundance and top two occupied less than $30 \%$. The remaining isoforms covered very small part in gene abundance. This result indicated that the genes with top one or top two abundance isoforms were major contributors for multi-isoforms. In order to reduce the false positive of computer predicting and effectively mine the functional isoforms, the expression threshold value of isoform was detected using the empirical method [30]. We identified 2.6 FPKM for each isoform with repeatedly detected in each biological replicate for further analysis (Additional file 2: Figure S2). Following the criteria, 58,909 isoforms with 29,368 high confidence unigenes were identified. Of the predicted genes, $46.13 \%(13,546)$ had two or more isoforms and $53.87 \%(15,822)$ had only one isoform. In addition, $81.51 \%$ of the isoforms $(48,019)$ were involved in 21,527 unigenes with two or more exons and $18.49 \%(10,890)$ had only one exon (Additional file 3: Figure S3). These multiisoforms genes or multi-exons isoforms have the potential to generate AS events. In addition, 40,016 isoforms were detected in RC group, 41,443 in RS group, 39,758 in LC group, and 40,572 in LS group. We found that the number of isoforms increased in both roots (3.57\%) and leaves (2.05\%) under salt stress (Table 1).

\section{Identification of alternative splicing events}

We used ASTALAVISTA to identify and classify the different types of alternative splicing. A total of
14,172 AS events were identified from 6798 genes in all 40 libraries, implying that nearly $31.58 \%$ of multiexonic genes $(6798 / 21,527)$ in roots or leaves underwent alternative splicing in G. davidsonii. We found that four basic AS types accounted for $85 \%$ of all AS events (Fig. 2a and Table 2). IR and ES events were the most and least frequent, and accounted for $35.73 \%$ (5063) and 7.71\% (1093) of cases, respectively. AA events (29.25\%) were more frequent than AD events (12.76\%). These results are consistent with those of previous reports $[5,16,31]$, which identified IR as the most frequent type of event in plant. Further, the distribution of four basic alternative splicing events in tissue- or treatment-specific groups were consistent with total events described above (Fig. 2b and Additional file 4: Table S1). As the

Table 1 Increased isoforms under salt stress in roots and leaves, respectively

\begin{tabular}{llll}
\hline Treatment & Isoform no. & Gene no. & Isoform per gene \\
\hline RC & 40,016 & 22,941 & 1.74 \\
RS & 41,443 & 23,658 & 1.75 \\
LC & 39,758 & 22,993 & 1.73 \\
LS & 40,572 & 23,294 & 1.74 \\
Total & 58,909 & 29,368 & 2.01 \\
\hline
\end{tabular}

$R C$ root well-watered control, $R S$ root salt-stressed treatment, $L C$ leaf wellwatered control, $L S$ leaf salt-stressed treatment 


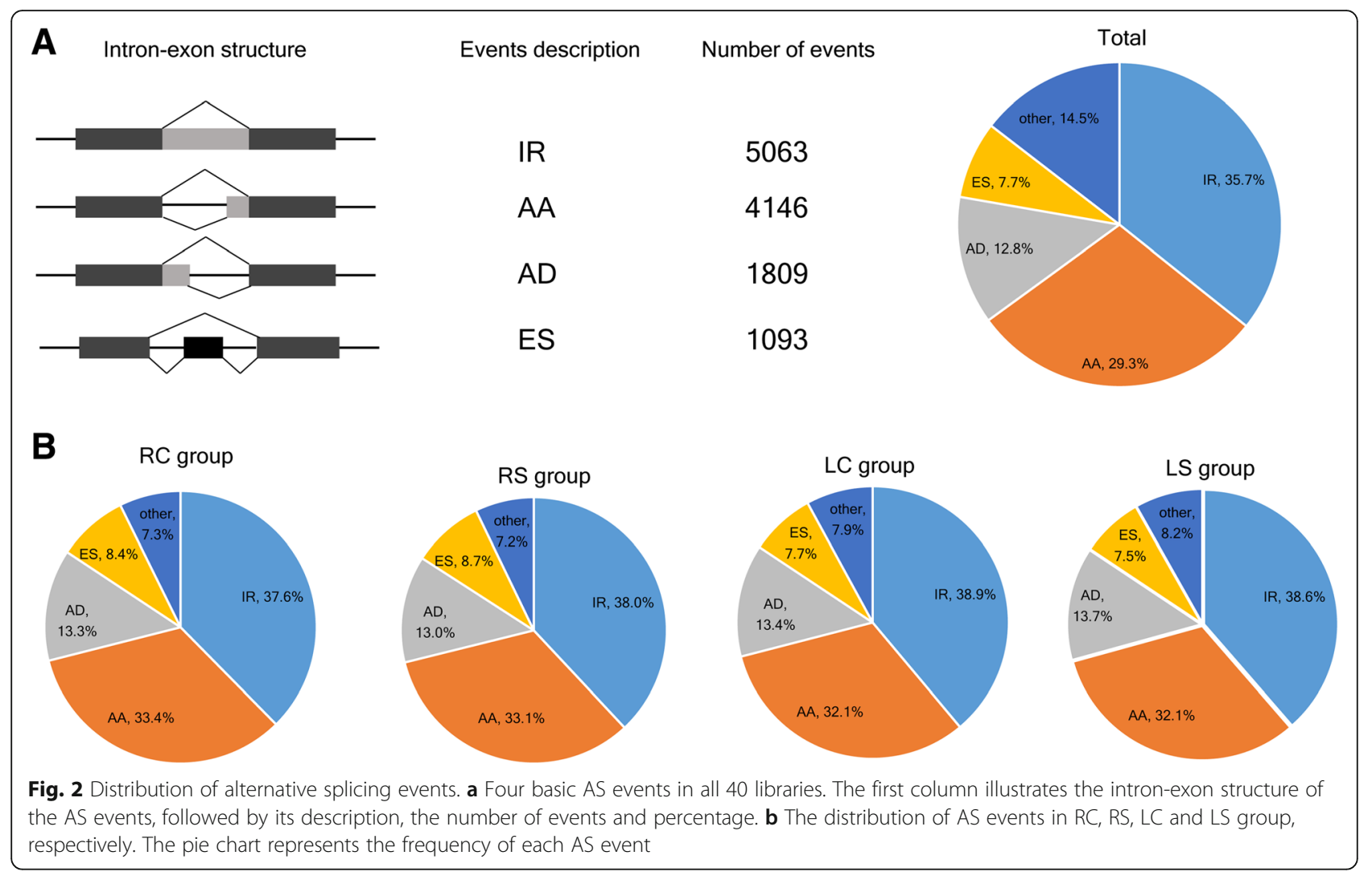

isoforms increased under salt stress condition (Fig. 3a), all four basic events were increased in both roots and leaves post salt stress (Fig. $3 \mathrm{~b}$ and Additional file 4: Table S1). It is consistent with the previous studies that AS was induced by abiotic stress [23, 32]. Only 2462 common events (17\% of total events) in four groups were detected, which implied that most events were tissue or salt stress specific (Fig. 4a). In detail, 5069 AS events were present in both leaf and root tissues, while 5035 and 5893 events were root- or leaf-specific (Fig. 4b). Eight thousand two hundred

Table 2 Number of AS events in total libraries

\begin{tabular}{llllll}
\hline Events $^{\mathrm{a}}$ & Events no. & Events rate & Gene no. & Gene rate $^{\mathrm{b}}$ & $\begin{array}{l}\text { AS per } \\
\text { gene }\end{array}$ \\
\hline $\mathrm{AA}$ & 4146 & $29.25 \%$ & 3065 & $45.08 \%$ & 1.35 \\
$\mathrm{AD}$ & 1809 & $12.76 \%$ & 1526 & $22.45 \%$ & 1.19 \\
$\mathrm{ES}$ & 1093 & $7.71 \%$ & 888 & $13.06 \%$ & 1.23 \\
IR & 5063 & $35.73 \%$ & 3492 & $51.37 \%$ & 1.45 \\
Other & 2061 & $14.54 \%$ & 1171 & $17.23 \%$ & 1.76 \\
Total & 14,172 & $100 \%$ & 6798 & $149.19 \%$ & 2.08 \\
\hline
\end{tabular}

${ }^{\mathrm{a}} A A$ alternative acceptor site, $A D$ alternative donor site, $E S$ exon skipping, $I R$ intron retention

${ }^{\mathrm{b}}$ The total gene rate is higher than $100 \%$ is due to one gene may undergo two or more AS events seventy-one AS events were present in tissues under both stress and control conditions, while 3618 and 4108 events were specific to normal and salt stress conditions, respectively (Fig. 4c). Therefore, AS events in G. davidsonii differed more between tissues than under stress treatment $(p<0.001$, Fisher's exact tests).

\section{Characteristics of different AS types}

We found that the event length of the different AS types showed divergence (Fig. 5a). For IR, the retained intron length ranged from 21 to $2771 \mathrm{bp}$, with the largest number of events at $83 \mathrm{bp}$, which was similar to soybean [22] and shorter than rice [33]. Our analysis also showed that the most frequent AA length was $3 \mathrm{bp}$, and the most frequent $\mathrm{AD}$ length was $4 \mathrm{bp}$, which is in agreement with previous findings from other species [22, 34, 35]. The skipping length in ES has multi-peaks, ranging from 20 events at $\sim 69 \mathrm{bp}, 22$ at $\sim 72 \mathrm{bp}, 22$ at $\sim 75 \mathrm{bp}$, and 19 at $\sim 84 \mathrm{bp}$. We also found both length of intron in IR events and exon in ES events were significantly $(p<0.01$, Student's $t$ test) shorter than the average length of intron and exon in total genome (Fig. 5b). In addition, according to the remainder when the length of events is divided by 3 , we group each event into three categories: splicing events with length of multiples of three nucleotides named as $\mathrm{AS}_{0}$, the remainder 1 as $\mathrm{AS}_{1}$ and the 

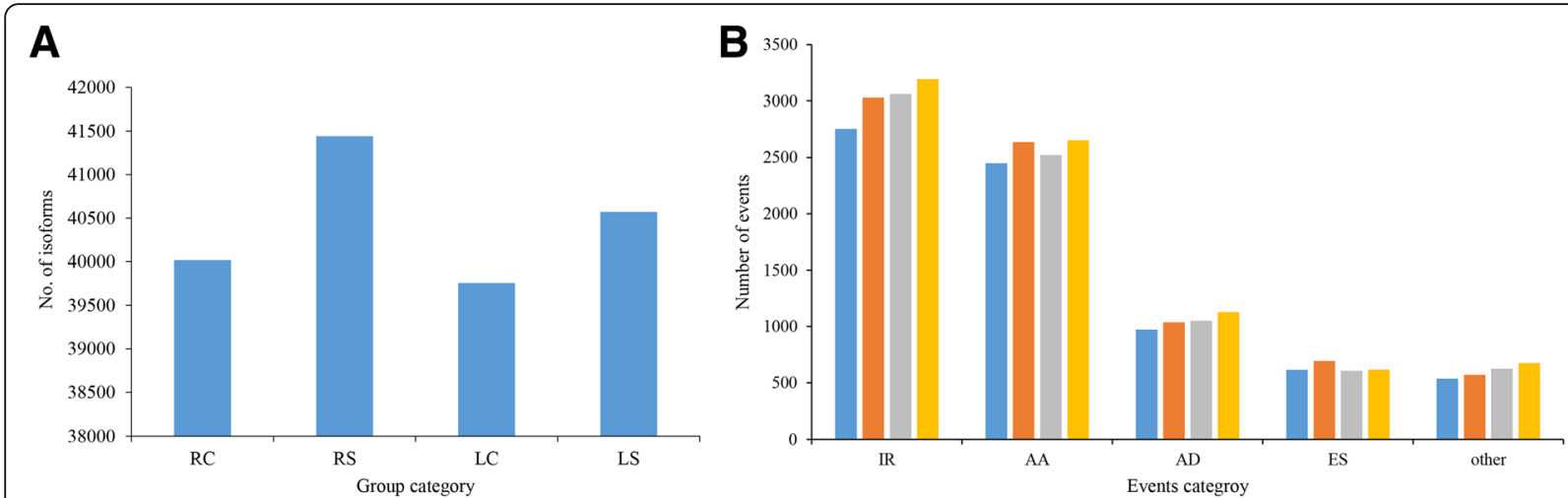

Fig. 3 Number of isoform and AS events in four groups. a The number of isoforms in four groups. $\mathbf{b}$ The number of AS events in four groups

remainder 2 as $\mathrm{AS}_{2}$. In general, splicing of $\mathrm{AS}_{1}$ or $\mathrm{AS}_{2}$ changes the reading frame, which either alters protein $\mathrm{C}$ termini or introduces premature termination codons (PTC) downstream from the splice junction, but splicing of $\mathrm{AS}_{0}$ does not. In total, $\mathrm{AS}_{0}$ type was dominant in four AS events and exon in total genome, except the intron (Fig. 5c). Especially, $41.8 \%$ in ES and $43.0 \%$ in AA were $\mathrm{AS}_{0}$ type, higher than $\mathrm{AS}_{1}$ and $\mathrm{AS}_{2}$ types, and the four peaks of ES length were all $\mathrm{AS}_{0}$ type. Even though each of three types in the intron accounted for $33.3 \%$ of total genome in average, all $\mathrm{AS}_{0}$ type of intron in IR events was $36.8 \%$ higher than the other two type which accounted for 31.5 and $31.7 \%$, respectively. The result implies that more AS events in cotton undergo evolutionary pressure to preserve the reading frame.

\section{Changes in splicing patterns associated with stress response}

To investigate the potential influence of salt-stressinduced AS on cellular processes, we analyzed functional categories related to genes with AS. Totally, we identified 1287 and 1228 differential alternative splicing events (DAS), involved in 808 genes in roots and 791 genes in leaves, under salt stress (Fig. 6a). The length distribution of these DAS events was generally consistent with the AS events described above (Additional file 5: Figure S4). And $\mathrm{AS}_{0}$ type was also dominant in four AS events, even occupied $84 \%$ of total DAS events in ES type (Additional file 6: Figure S5). To determine whether the DAS events were affected by gene transcription, we compared the DAS genes with the differential expressed

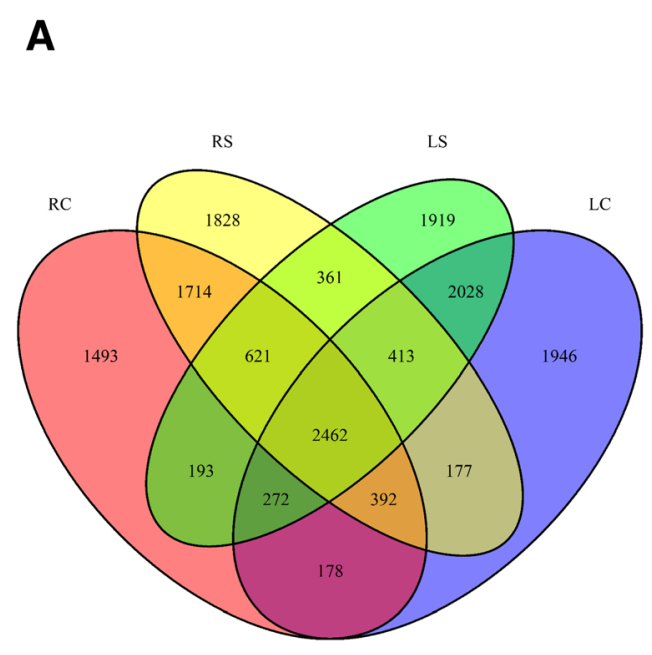

B

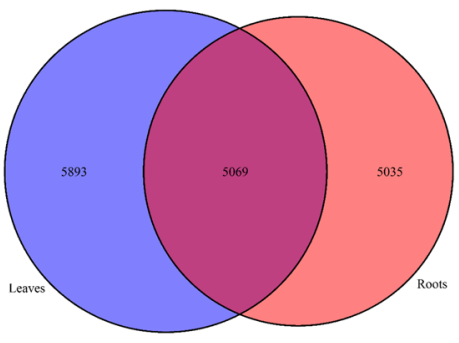

C

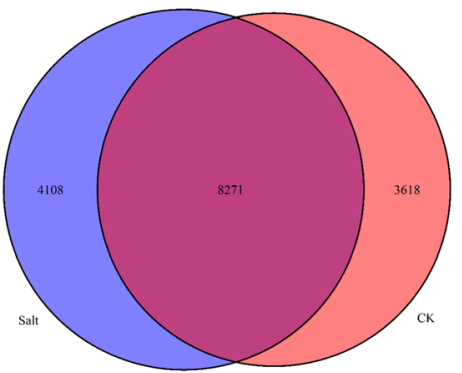

Fig. 4 Venn diagram of alternative splicing events in roots and leaves at normal and salt stress condition. a Venn diagram of the overlap of alternative splicing events in four groups. b The common and specific alternative splicing events between roots and leaves. $\mathbf{c}$ The common and specific alternative splicing events between well-watered and salt stress condition 


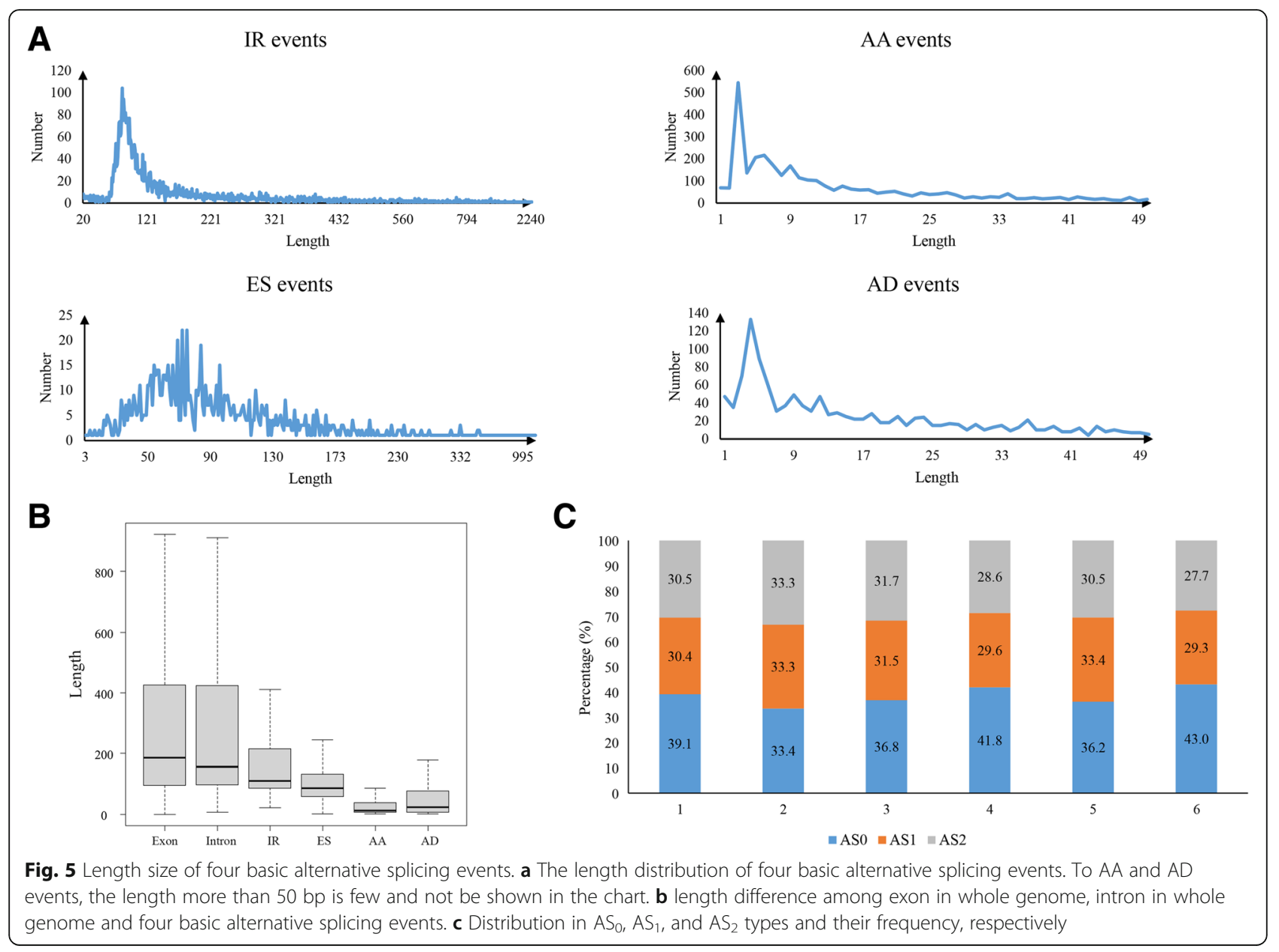

genes identified in our previous study [26]. Only a small subset of DAS genes $(18.02 \%$ in roots and $21.63 \%$ in leaves) overlapped with the differential expressed genes (Additional file 7: Figure S6). This result suggests that the transcriptional activity of genes induced by salt stress has no significant effect on DAS events, with the similar report in Physcomitrella patens [24].

Based on the Gene Ontology (GO) analysis, we found that the differential alternative splicing overrepresentation of different $\mathrm{GO}$ categories in both roots and leaves, and the number of isoforms involved in related terms increased under salt stress (Fig. $6 \mathrm{~b}$ and Additional file 8: Table S2). Most terms were common enriched in roots and leaves, such as "response to salt stress", "water transport" and "alcohol catabolic process". In addition, photosynthesis related terms in leaves, such as "photosynthesis" and "chlorophyll metabolic process", were exclusively overrepresented. Although most biological process were commonly overrepresented in roots and leaves, tissue-specific regulation of DAS was distinct, and less than 20\% DAS genes were simultaneously detected in the two tissues (Fig. 6a).
We found 81 DAS events involved in 58 genes in "response to salt stress" terms (Additional file 9: Table S3). Most of them were regulated by IR and AA events. The expression of AS events was various under salt stress, with some increased and the other decreased (Fig. 7). We also performed the correlation analysis between AS frequency change values and fold change values of gene expression for salt response genes (Additional file 10: Table S4). The results showed that only 5\% (4/81) DAS events were significantly correlated with their gene expression, implying that AS acts an independent regulatory pattern comparing to transcription regulation under salt stress. In the DAS genes, we found that some important genes involved in the response to salt stress, such as Pyrroline-5-carboxylate synthase (P5CS, Gorai.012G107700), $\mathrm{K}^{+}$channel outward (KCO1, Gorai.013G153400) and plasma membrane intrinsic protein (PIP, Gorai.011G098100), generated novel AS isoforms. P5CS plays crucial roles in the proline biosynthesis pathway [36] and proline is an important compatible solute for osmosis homeostasis under salt stress [37]. KCO1 is involved in the $\mathrm{K}^{+}$ 


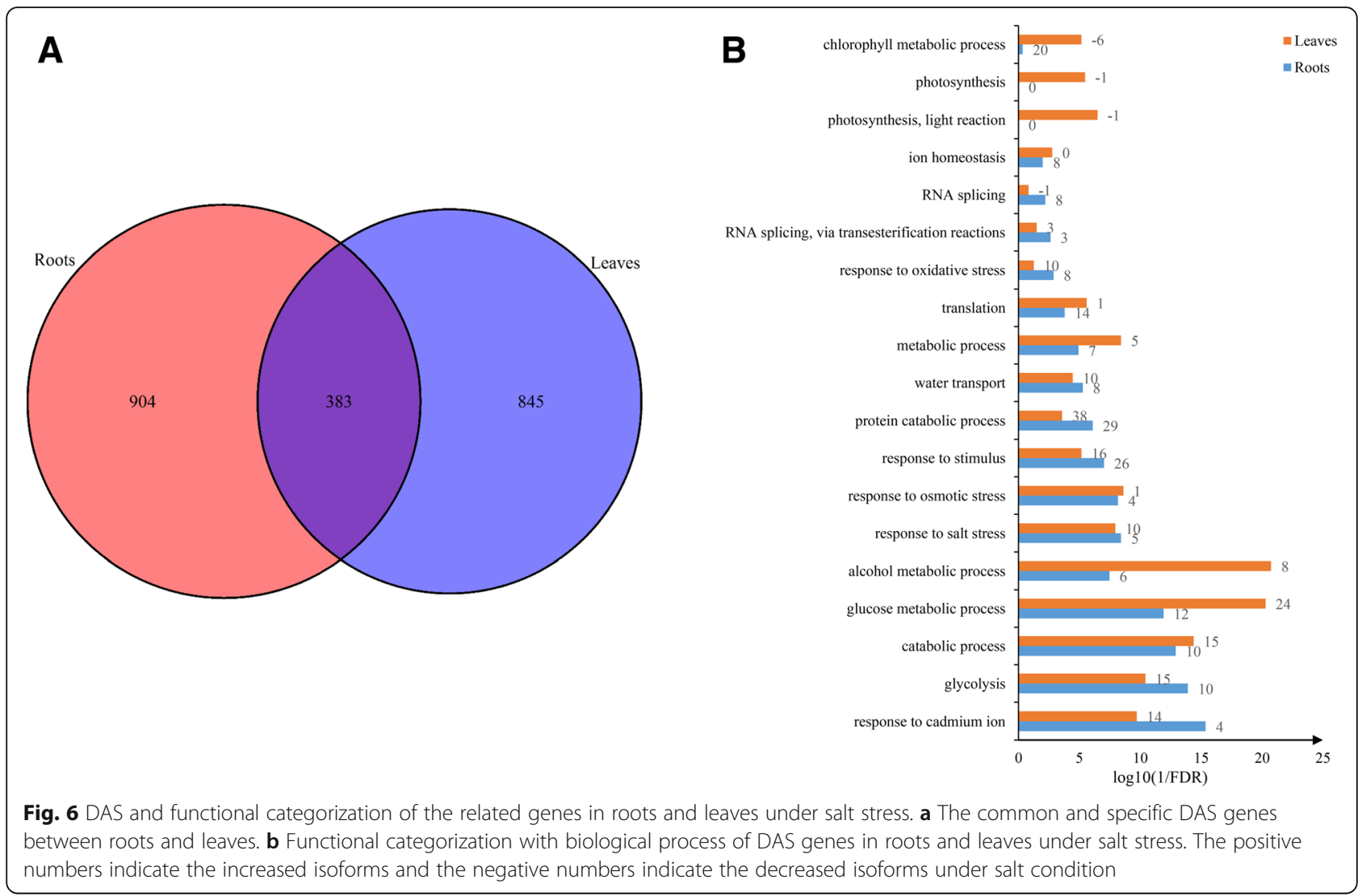

transport, which contributes to ion homeostasis [38]. PIP is a member of the aquaporin family, which is a crucial water channel protein involved in the salt stress response [39]. In addition, we also found some transcription factors that were related to the salt stress response, such as WRKY (Gorai.012G119600) [40], MYB (Gorai.009G288900) [41] and bHLH (Gorai.009G396900) [42]. Taken together, there exists the complex regulating mechanism in response on salt stress in cotton.

\section{Splicing factors involved in AS under salt stress}

RNA splicing genes, especially those encoding splicing regulators, are known to be actively alternatively spliced under stress conditions [43]. The serine/arginine-rich (SR) proteins are known to be involved in pre-mRNA splicing processes and regulate alternative splicing by changing the splice site selection in a concentration dependent-manner [44]. In this study, 29 SR orthologous genes (Additional file 11: Table S5) were identified in the G. raimondii annotation information (http://genome.jgi.doe.gov/). Of them, 13 (12 in roots and seven in leaves) were differentially alternatively spliced under salt stress (Table 3). However, only two SR orthologous genes (Gorai.009G393200, Gorai.010G201600) were differential expression (up-regulated) under salt stress [26], indicating that SR genes were more inclined to be regulated by post- transcriptional level instead of transcriptional level. Following the Arabidopsis SR protein family divided into six subfamilies [25], we found that most differential splicing SR proteins in cotton belonged to RSZ subfamily, which was not reported in Arabidopsis, implying the different splicing regulation under salt stress between in cotton and Arabidopsis.

\section{Validation of predicted AS and DAS events}

In order to evaluate the reliability of computationally predicted AS events, nine AS events with the length ranging from 60 to $200 \mathrm{bp}$ for detection by agarose gel analysis, were randomly selected and validated by RTPCR using intron-flanking primers (Additional file 12: Figure S7 and Additional file 13: Table S6). As a result, the PCR products were more than one in agarose gel corresponding to the AS events. Meanwhile, we also found the amplification products difference in different tissues or treatments, implying that AS was complicated and sensitive in different conditions. Further, ten DAS genes, which included three salt stress response genes reported previously and seven other induced genes, were validated by evaluating the relative abundance of the splicing event by qRT-PCR (Fig. 8, Additional file 14: Table S7 and Additional file 15: Figure S8), and the ratio between normal condition and salt condition for each 


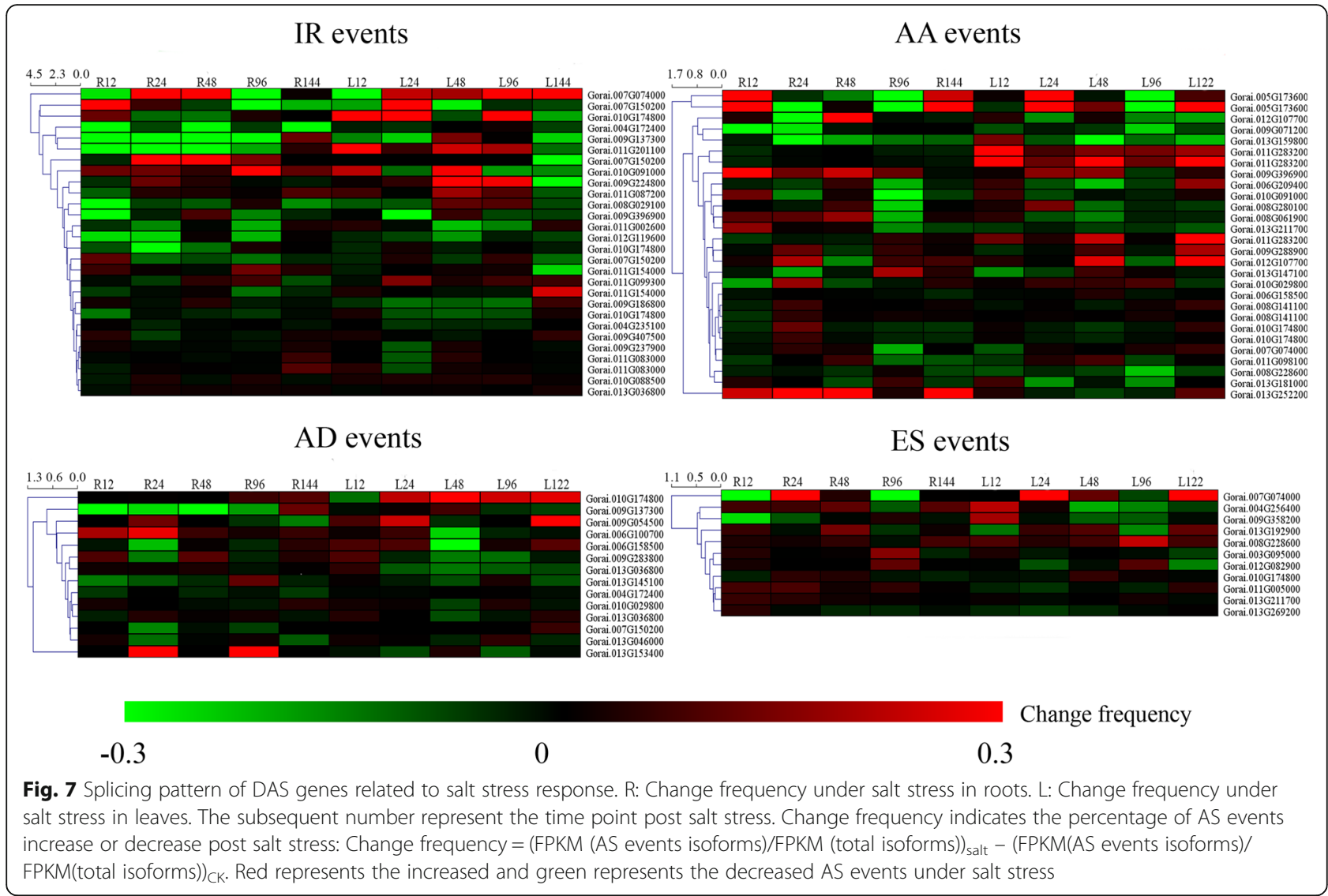

variant. In total, $80 \%(8 / 10)$ predicted events were able to be detected by qRT-PCR. The events that were unable to be validated may be the result of false computational predictions or low transcript expression. A plasma membrane intrinsic protein (GrPIP2.7, Gorai.011G098100) had three isoforms. GrPIP2.7_t1 was the dominant isoform that highly expressed in both roots and leaves, followed by GrPIP2.7_t2 and GrPIP2.7_t3. GrPIP2.7_t3 was generated by an AS event found at an alternative 3' acceptor site (AA) which introduced a PTC at the MIP domain on the third exon. RNA-seq data and qRT-PCR revealed that the proportion of T3 increased under salt stress (Fig. 8a). $\mathrm{K}^{+}$channel outward (GrKCO1, Gorai.013G153400) had two isoforms. GrKCO1_t1 was the dominant isoform and an alternative $5^{\prime}$ donor site (AD) located on the first exon within $5^{\prime}$ UTR region that would not change the integrity of CDS region. RNA-seq data and $\mathrm{qPCR}$ revealed that the proportion of GrKCO1_t 2 increased under salt condition (Fig. 8b). A WRKY transcription factor (GrWRKY33, Gorai.012G119600) had four isoforms. The gene had four introns and GrWRKY33_t1 was the dominant isoform. IR event was detected in GrWRKY33 t2, GrWRKY33 t3 and GrWRKY33_t4 on the second, third and fourth intron, respectively. Among these three IR events, only the last one was detected differential splicing and would be used to verify, however, the other two would not. IR in GrWRKY33 t3 and GrWRKY33_t4 introduced PTC caused the second function motif lost. Different from the former, IR in GrWRKY33_t2 introduced PTC at the $5^{\prime}$ CDS region but not affected the dominant function. RNA-seq data and qRT-PCR revealed that IR event on the fourth introns was decreased under salt stress (Fig. 8c), indicating that salt stress elevated the proportion of function isoforms of GrWRKY33.

\section{Discussion}

Plants require controlled systems to defend against various stresses. Previous studies have reported many genes that are responsive to stresses at the transcriptional level $[45,46]$, however, gene regulation at the posttranscriptional level was less investigated under stress, especially for salt stress. Since Berget et al. [47] discovered intervening sequences, an increasing amount of evidence has revealed that AS plays an important role in transcription regulation and contributes to the functional diversity of eukaryotic genomes. AS is involved in many physiological processes, including the responses to biotic and abiotic stresses $[12,15,16]$. Most of the AS events that have been found to be involved in the response to abiotic stress are linked to genes with regulatory roles, covering all 
Table 3 The components of splicing machinery involved in regulation of salt stress responses in $\mathrm{G}$. davidsonii

\begin{tabular}{lllll}
\hline ID in G. raimondii & ID in A. thaliana & Gene symbol & RDAS & LDAS \\
\hline Gorai.010G203100 & AT3G53500 & RSZ32 & AA & AA \\
Gorai.011G242700 & AT3G53500 & RSZ32 & AA & \\
Gorai.013G024100 & AT3G53500 & RSZ32 & AA & AA \\
Gorai.004G044100 & AT3G61860 & RSP31 & AA & \\
Gorai.002G165700 & AT4G31580 & SRZ22 & IR & AA \\
Gorai.010G121100 & AT4G31580 & SRZ22 & ES & AA \\
Gorai.010G245400 & AT4G31580 & SRZ22 & IR & IR \\
Gorai.005G220000 & AT5G64200 & SC35 & IR & IR \\
Gorai.007G128300 & AT1G02840 & SRP34 & Other & \\
Gorai.002G252500 & AT2G37340 & RSZ33 & IR & \\
Gorai.012G091800 & AT2G37340 & RSZ33 & AA & \\
Gorai.010G126300 & AT3G13570 & SCL30A & & ES \\
Gorai.008G280300 & AT4G25500 & ATRSP40, RSP35 & AD & \\
\hline
\end{tabular}

RDAS differential alternative splicing types in roots, LDAS differential alternative splicing types in leaves

levels of the regulation of gene expression [48]. Nevertheless, large-scale or genome-wide studies of AS dynamics under salt stress conditions are still relatively scarce. In this study, through comprehensive transcriptome analysis of high-throughput RNA-seq data, we revealed genome-wide AS events under salt stress in G. davidsonii. Our analysis suggests that $31.58 \%$ of the multi-exonic genes in the G. davidsonii genome are alternatively spliced under salt-stress conditions. Furthermore, four basic AS events was increased post salt stress, and we have identified DAS genes associated with several biological processes, such as "response to salt stress", "water transport" and "metabolic process". Moreover, we observed that genes encoding splicing factors are frequently alternatively spliced under salt stress.

\section{An overview of AS in G. davidsonii under salt stress}

In G. raimondii, 16,437 AS events involving 77,569 unique transcripts at 10,197 genes have been identified, indicating that $\sim 32 \%$ of the multi-exonic genes had at least one AS event [7]. In this study, 14,172 AS events involving 6798 genes were identified in G. davidsonii suggested that $31.58 \%$ of the multi-exonic genes were alternatively spliced. The ratio of alternative splicing genes is lower than other plants $[22-24,30]$ suggests difference between cotton and other plants. We also found that IR was less frequent and AA was more frequent in G. davidsonii compared with that in G. raimondii, implying interspecific differences in the types of AS events in the two diploid D-genome cotton species. Actually, the differences in alternative splicing have also been described between different ecotypes of Arabidopsis [49] and Vitis vinifera [31], giving support to the argument that changes in splicing may contribute to the evolutionary adaptation process. Furthermore, most AS events were tissue or stress specific, only $17 \%$ of total events were commonly detected in all four tissues/treatments conditions in G. davidsonii (Fig. 3a). Similar AS profiles are also observed in other plants, such as Arabidopsis [23], Vitis vinifera [31] and Zea mays [50]. The result implied that AS may contribute to the function diversity in the tissue or stress-specific genes.

\section{AS was regulated in response to salt stress}

We found that AS events were increased under salt stress no matter in roots or in leaves. This finding is consistent with the previous studies on AS under environmental stresses [23, 48]. These increased AS events may lead to a wider plasticity for plants to enable them to adapt to various stresses. To further understand the gene function affected by alternative splicing under salt stress, we performed gene ontology (GO) analysis of DAS and found that they were enriched in some biological processes, such as "response to salt stress", "water transport" and "metabolic process". This result further suggested that the DAS induced by salt stress could regulate the salt response. An interesting finding is that "response to calcium ion" is not only overrepresented in our study, but also frequently overrepresented in other plants [23] or other stress condition [32]. Calciumsignaling pathway plays a crucial role in stress response. Exposure to salinity activates the Salt Overly Sensitive (SOS) pathway, leading to $\mathrm{Ca}^{2+}$-dependent increased activity of SOS1, a plasma membrane $\mathrm{Na}^{+}-\mathrm{H}^{+}$antiporter that enables adaptation through $\mathrm{Na}^{+}$efflux $[51,52]$. We suspect that Calcium regulation pathway is sensitive to stress condition at splicing level. Some important salt stress response genes or transcript factors, such as KCO, PIP and WRKY, were regulated by AS. The expression of these genes may have three fates, that decreased the abundance of function isoforms by upregulated the alternative splicing isoforms like GrPIP2.7, that increased the abundance of function isoforms by downregulated the alternative splicing isoforms like GrWRKY33, and that remained the abundance with AS events occur at $5^{\prime}$ UTR region that may not affect the translation of CDS region like GrKCO1. In addition, DAS genes induced by salt stress were enriched in pathways related to RNA splicing, indicating that AS events of the RNA splicingrelated genes are induced by salt stress and regulated by themselves or other splicing factors [53, 54]. These AS events may introduce new domain and subsequently impact the function of gene $[55,56]$. Consequently, AS is a complex and important regulatory mechanism in response to salt stress in cotton. 


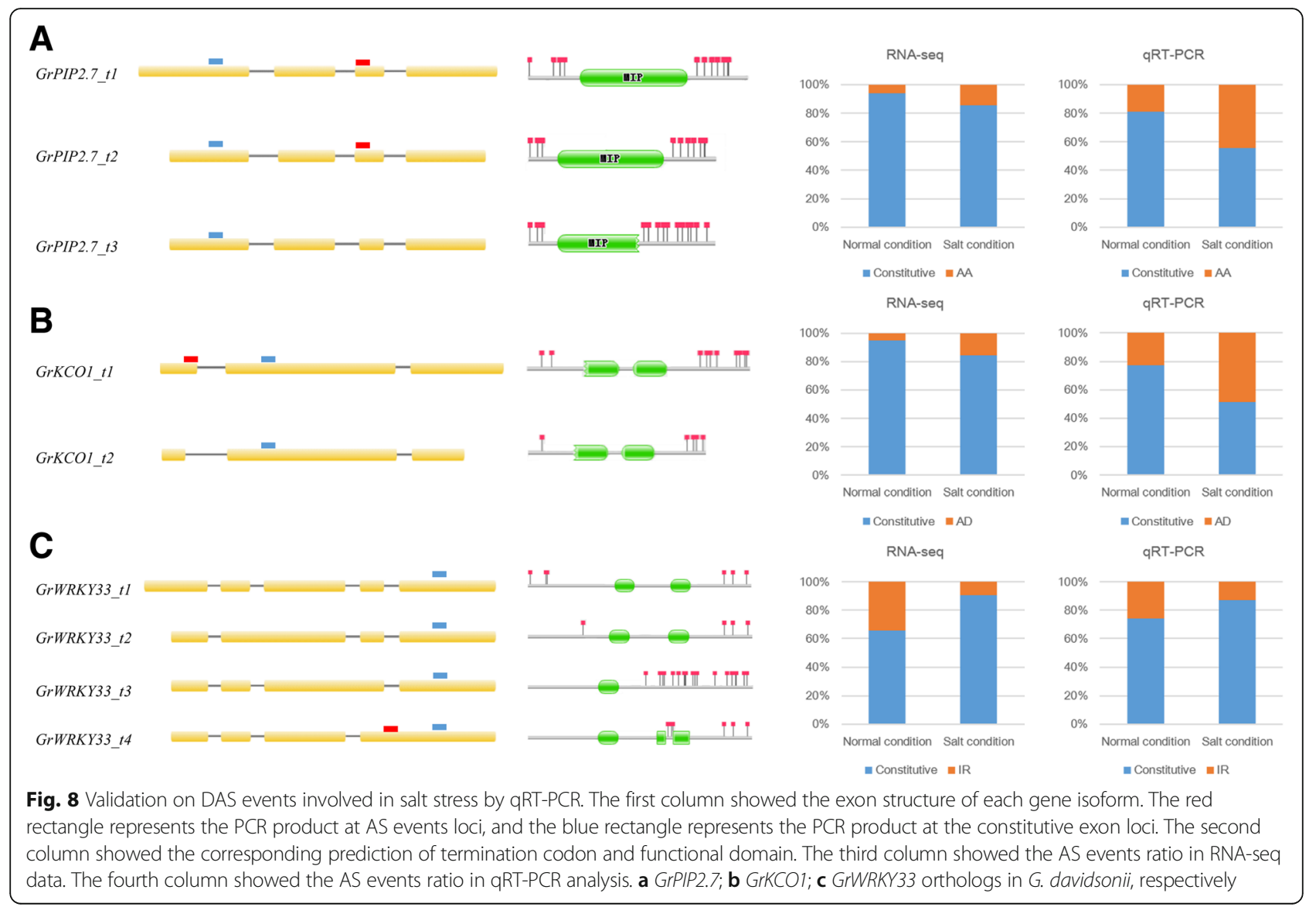

SR splicing factors were regulated by AS under salt stress The splicing of introns from pre-mRNA is carried out by one of the largest molecular complexes of the cell, the spliceosome, which consists of five small nuclear ribonucleoproteins (snRNPs) and numerous additional proteins [10]. Members of the Serine/arginine (SR) protein family are well-known non-snRNP spliceosomal factors [57]. A few previous studies showed that overexpression of SRs or other splicing factors could increase plant tolerance to salt and other stresses [43, 58-60]. Interestingly, the AS pattern of most SRs has been shown to change under stress conditions [12, 23]. It suggests that pre-mRNAs of SRs are themselves alternatively spliced and this splicing is under tight spatial, temporal, and environmental control. In this study, 29 orthologous SR genes were identified in G. davidsonii. Of them, 16 were differential splicing and only two were differential expression in roots or leaves. This result is consistent with the previous report on Arabidopsis [23], which show that splicing factors involved in salt stress function mainly via splicing pathways rather than regulated directly by salt stress. Thatcher et al. [30] also demonstrated that the expression level of known splicing factors was not the major driving force behind genotypic AS variation. Therefore, we speculated that pre-mRNAs of SRs were themselves alternatively spliced under salt stress and the diversity of SR splicing products consequently increased the number of novel AS isoforms identified (Figs. 3a and 6b), and SR genes belong to RSZ subfamily might play a crucial role in splicing regulation under salt stress condition in cotton.

\section{AS plays a crucial role in salt stress response at the posttranscriptional level}

The molecular mechanisms of the response to salt stress involve signal transduction pathways [61], transcription factors [62] and genes [63], which have been well documented at the transcriptional level. However, little is known about the regulation of salt stress-specific gene expression at the post-transcriptional level. Here, we found that AS events were abundant in the response to salt stress in G. davidsonii. However, one crucial question is whether the increase in AS events is an acclimation response or merely a consequence of splicing errors caused by stress damage. Several previous studies suggested that the stress-induced increase in AS could be ascribed to splicing errors and could weaken the function of the corresponding genes by decreasing the abundance of functional transcripts $[23,64]$. However, some evidence showed that AS could promote stress tolerance 
by increasing proteomic diversity $[65,66]$. Most aberrant splicing events (splicing errors) could be removed by mRNA surveillance mechanisms such as nonsense mediated mRNA decay (NMD) [67], thus, some splicing variants, neutral or beneficial to the organism, can be selectively fixed as functional AS events. A large-scale study using 39 million expressed sequence tags from 47 eukaryotic species revealed that the proportion of AS genes and the average number of AS isoforms per gene (AS level) have gradually increased over the past 1.4 billion years, indicating that AS complexity can be considered a strong predictor of organismal complexity [68]. In present study, we found the number of AS events and isoforms increased under salt stress condition (Figs. 3 and 6). In addition, DAS genes were overrepresented in terms of "response to salt stress" and "metabolic process". We also found that the splicing pattern was diverse in tissues and developmental stage under salt stress (Fig. 7). Compared to the differential expression regulation in our previous study [26], salt stress response at splicing level is more complicated. The specific regulation pathway of response to salt stress is hard to verify only by transcriptome sequence, and further research at molecular level, based on the key genes regulated at splicing level such as P5CS and PIP, need to be conducted.

\section{Conclusion}

In this study, through comprehensive transcriptome analysis of high-throughput RNA-seq data, we revealed genome-wide AS events under salt stress in G. davidsonii. We found that that the number of AS events under stress conditions was significantly higher than that under normal conditions. The functions of these genes related to AS were enriched in several biological processes, such as "response to salt stress", "water transport" and "metabolic process". We also detected splicing factors (SFs) involved in AS regulation and found their alternative splicing under salt stress. In addition, several salt response genes, such as P5CS, KCO1, PIP and WRKY33, were identified with differential splicing. This study indicates that AS plays the important roles in plant response to salt stress.

\section{Methods}

\section{Plant material and salt stress conditions}

Diploid wild cotton species G. davidsonii was used for the study. Following our pervious study, the salt stress concentration of $200 \mathrm{mM} \mathrm{NaCl}$ was identified by comparing the salt tolerance of three cotton accessions, diploid wild cotton species G. davidsonii and two G. hirsutum cultivars, ZS9612 and Z9807, with sensitivities and insensitivities to salinity stress, respectively [26]. All necessary permits for collecting the G. davidsonii seeds were obtained from Nanjing Agricultural University, China.

The G. davidsonii seeds were surface-sterilized with $70 \%$ ethanol for $30-60$ s and $10 \% \mathrm{H}_{2} \mathrm{O}_{2}$ for $60-120 \mathrm{~min}$, followed by washing with sterile water. Sterilized seeds were germinated at $26^{\circ} \mathrm{C}$ under long day conditions in a $16 \mathrm{~h}$ light $/ 8 \mathrm{~h}$ dark cycle with a light intensity of $150 \mu \mathrm{mol} \mathrm{m}^{-2} \mathrm{~s}^{-1}$ on $1 / 2 \mathrm{MS}$ solid medium. Three days after germination, the plants were transferred to $1 / 2$ Hoagland nutrient solutions at $\mathrm{pH}$ 6.0.

The seedlings with two true leaves and one heartshaped leaf were randomly selected and cultured in $1 / 2$ Hoagland solutions supplemented with $200 \mathrm{mM} \mathrm{NaCl}$ for salinity stress treatment. Due to different salt damage mechanisms when plant exposure to salinity [69, 70], five time points, 12, 24, 48, 96, and $144 \mathrm{~h}$ after exposure, were set for the leaf and root sampling. All samples were frozen quickly in liquid $\mathrm{N}_{2}$ and stored at $-70{ }^{\circ} \mathrm{C}$ for further use. Plant seedlings grown in normal 1/2 Hoagland nutrient solution was used as controls. All the cotton plants cultured in $1 / 2$ Hoagland solutions were grown in chambers under long day conditions with a $16 \mathrm{~h}$ light/ $8 \mathrm{~h}$ dark cycle at $28 / 25{ }^{\circ} \mathrm{C}$ in Nanjing Agricultural University. The 1/2 Hoagland nutrient solution was replaced every day. These evaluations were not relevant to human subject or animal research. Therefore, they did not involve any endangered or protected species.

\section{RNA extraction, CDNA library preparation, and RNA-seq}

Total RNA was extracted from roots and leaves by the cetyltrimethylammonium bromide (CTAB)-sour phenol extraction method [71]. The RNA was digested with RNase-Free DNase (Qiagen) and checked for integrity by capillary gel electrophoresis. Library preparation for RNA-seq was performed using the TruSeq RNA Sample Preparation Kit (Illumina, Cat. NRS-122-2002) with $500 \mathrm{ng}$ of total RNA. Accurate quantitation of cDNA libraries was performed using the QuantiFluor dsDNA System (Promega). The size range of the final cDNA libraries was determined by applying the DNA 1000 chip on the Bioanalyzer 2100 (Agilent; $280 \mathrm{bp}$ ). The cDNA libraries were amplified and sequenced using the cBot and HiSeq2000 systems from Illumina. Two biological replicates from each sample were used for all RNA-seq experiments.

\section{Reads mapping and transcript assembly}

After preprocessing the RNA-seq data with an NGS QC toolkit [72], the reads were mapped to the G. raimondii genome using a Tophat spliced aligner [73]. Based on default Tophat parameters, four mismatches were allowed for reads mapping. The sequence alignment/map files generated by Tophat were used as the input to the software Cufflinks [74], which assembles the alignments in the 
sequence alignment/map file into transfrags. Cufflinks does this assembly independently of the existing gene annotations and constructs a minimum set of transcripts that best describes the RNA-seq reads. The unit of measurement used by Cufflinks to estimate transcript abundance is FPKM. The Cufflinks statistical model probabilistically assigns reads to the assembled isoforms. Cuffcompare was used to merge the assemblies with the reference annotation (G.raimondii_221_v2.1.gene.gff) into a single GTF file that was used later to identify alternative splicing (AS) events. The class codes in the Cuffcompare output were used to identify novel isoforms, intergenic transcripts, and splice junctions.

\section{Novel isoforms prediction and alternative splicing analysis}

Using an empirical method [30], 2.6 FPKM was chosen as the expression cutoff for alternative splicing isoforms. In addition, the AS isoforms that were identified in both replications, were regarded as the stable isoforms. We used the ASTALAVISTA v2.2 software [75] with the parameters (-t asta -i) to quantify the type of AS events based on the assembled transcripts by the Cufflinks software. Four basic AS events (IR, AA, AD and ES) were performed and the remaining complex AS events were collectively grouped as other type.

\section{Differential alternative splicing events analysis}

Each AS event consist of constitutive and alternative splicing event (e.g. IR event consist of intron splicing and intron retain). First, FPKM of constitutive and alternative splicing event were calculated by sum of the corresponding isoforms (constitutive or alternative splicing event may have multiple isoforms). Then, fisher's exact test was applied to above FPKM to analyze differential alternative splicing between wellwatered and salt-stress treatments with $p<0.05$ as significance. In order to assess the change of AS events caused by salt stress, each FPKM of event was then converted into a percentage of total (FPKM (AS events isoforms)/FPKM (total isoforms)) and subsequently calculated the difference.

\section{Go enrichment}

AgriGO software [76] was used for gene ontology analysis, and Singular Enrichment Analysis (SEA) was performed with the statistical method of Fisher's exact tests. The input sample list was the G. raimondii gene ID (http://www.phytozome.net/), which was converted from the original ID of the Cuffdiff default configuration, and the background was whole annotated genes in G. raimondii. The output of enrichment needed Benjamini and Hochberg-adjusted $P$-values (FDR) $<0.05$.

\section{RT-PCR and $\mathrm{qRT}$-PCR validation}

RT-PCR and qRT-PCR were done using a new set of RNAs extracted with the same tissues and treatment time points as that for RNA-seq analysis. The selected AS events were validated by RT-PCR using a set of primers (Table S6) that were designed based on each AS event. EF1- $\alpha$ was used as an internal standard. The amplification reactions were performed under the following conditions: $95{ }^{\circ} \mathrm{C}$ for $5 \mathrm{~min}$, followed by 32 cycles of $95{ }^{\circ} \mathrm{C}$ for $30 \mathrm{~s}$, $58{ }^{\circ} \mathrm{C}$ for $30 \mathrm{~s}$, and $72{ }^{\circ} \mathrm{C}$ for $60 \mathrm{~s}$. qRT-PCR validations was performed on three alternative splicing events in the salt stress response genes, using a Bio-Rad CFX96 RealTime instrument and the light cycler fast start DNA Master SYBR Green I kit (Roche, Basel, Switzerland). Relative abundance of each splicing event by qRT-PCR using primers specific for each splicing variant or common primers for whole transcripts (Table S7). The GhHis3, which is a constitutive expression gene in cotton [77], its orthologous gene GrHis3 in G. raimondii was used to design the internal control primers (Table S7) and $\Delta \Delta \mathrm{Ct}$ method was used for data analysis [78].

\section{Additional files}

Additional file 1: Figure S1. Frequency of the top five most abundant isoforms across the samples. Each line in chart represent a sample with two biological replicates: the roots of well-watered control plants (RC), the roots of salt-stressed plants (RS), the leaves of well-watered controls plants (LC), the leaves of salt-stressed plants (LS) and the subsequent number represent the time point post treatment. (TIFF $452 \mathrm{~kb}$ )

Additional file 2: Figure S2. FPKM cutoff used for novel transcripts selection. The loss of expression of known transcripts (false negatives) plotted against the retention of randomly generated artificial transcripts (false positive) at various FPKM (1-10) abundance cutoffs. Fractions represent the number of isoforms found above a given cutoff in at least one library. (TIFF 130 kb)

Additional file 3: Figure S3. The numbers of exon and isoform distribution for high confidence unigenes. A. Number of exons per isoform distribution. B. Number of isoforms per gene distribution. (TIFF $120 \mathrm{~kb}$ )

Additional file 4: Table S1. Number of AS events in four groups. (DOCX $14 \mathrm{~kb}$ )

Additional file 5: Figure S4. The length distribution of differential alternative splicing events. To AA and AD events, the length more than $50 \mathrm{bp}$ is few and not be shown in the chart. (TIFF $341 \mathrm{~kb}$ )

Additional file 6: Figure S5. The length distribution of differential alternative splicing events. To $A A$ and $A D$ events, the length more than $50 \mathrm{bp}$ is few and not be shown in the chart. (TIFF $99 \mathrm{~kb}$ )

Additional file 7: Figure S6. The comparison of differential alternative splicing and differential expression genes. RDAS for differential alternative splicing in roots; RDEG for differential expression genes in roots; LDAS for differential alternative splicing in leaves; LDEG for differential expression genes in leaves. (TIFF 299 kb)

Additional file 8: Table S2. Biological process of DAS genes and their corresponding isoforms distribution. (DOCX $15 \mathrm{~kb}$ )

Additional file 9: Table S3. DAS genes related to salt stress response. (DOCX $17 \mathrm{~kb}$ )

Additional file 10: Table S4. Correlation analysis between frequency change of AS events and fold change of gene expression for salt response genes. (XLSX $32 \mathrm{~kb}$ ) 
Additional file 11: Table S5. Information on SR proteins identified in G. davidsonii. (DOCX $14 \mathrm{~kb}$ )

Additional file 12: Figure S7. RT-PCR validation of alternative splicing events. Each gene was amplified at the roots of well-watered control, the roots of salt-stressed, the leaves of well-watered controls and the leaves of salt-stressed condition. EF1-a was used as an internal standard. (TIFF $279 \mathrm{~kb}$ )

Additional file 13: Table S6. Primers used for RT-PCR of alternative splicing events. (DOCX $14 \mathrm{~kb}$ )

Additional file 14: Table S7. Primers used for qRT-PCR of differential alternative splicing events. (DOCX $15 \mathrm{~kb}$ )

Additional file 15: Figure S8. Validation on DAS events by GRT-PCR. The gene IDs marked in red indicates that the predicted DAS events are unable to be validated. His 3 is a constitutive expression gene in cotton, and GrHis3 (Gorai.003G041300) in G. raimondii was used to design primers for the internal control analysis. (TIFF $501 \mathrm{~kb}$ )

\section{Abbreviations}

AA: Alternative acceptor site; AD: Alternative donor site; AS: Alternative splicing; DAS: Differential alternative splicing; DEG: Differential expression genes; ES: Exon skipping; FPKM: Fragments per kilobase per million reads; GO: Gene Ontology; hnRNP: heterogeneous nuclear ribonucleoprotein particle; IR: Intron retention; LC: The leaves of well-watered controls plants; LS: The leaves of salt-stressed plants; NMD: Nonsense mediated mRNA decay; PTC: Premature termination codons; RBPs: RNA binding proteins; RC: The roots of well-watered control plants; ROS: Reactive oxygen species; RS: The roots of salt-stressed plants; SFs: Splicing factors; snRNPs: small nuclear ribonucleoprotein particles; SOS: Salt overly sensitive; SR: Ser/Arg-rich; UV: Ultraviolet

\section{Acknowledgements}

The authors would like to thank Dr. Caiping Cai from College of Agriculture in Nanjing Agricultural University for helpful comments.

\section{Funding}

This program was financially supported in part by the National Transgenic Program (2016ZX08005-004), Key R \& D program in Jiangsu Province (BE2015360), Six talent peaks project in Jiangsu province (2015-NY-002), the Fundamental Research Funds for the Central Universities (KYYJ201701) and JCIC-MCP project (No.10).

\section{Availability of data and materials}

RNA-seq data in this study have been deposited at the National Center of Biotechnology Information (NCBI, http://www.ncbi.nlm.nih.gov/) under the accessions SRP061663.

\section{Authors' contribution}

Experiments were designed by WZG. Experiments were performed by GZZ, WXL and FZ. GZZ and WZG drafted the manuscript, WZG revised the manuscript. All authors read and approved the final manuscript.

\section{Ethics approval and consent to participate}

Diploid wild cotton species $G$. davidsonii was used in the study. All necessary permits for collecting the G. davidsonii seeds and seedlings were obtained from Nanjing Agricultural University, China.

\section{Consent for publication}

Not applicable.

\section{Competing interests}

The authors declared that they had no competing interests.

\section{Publisher's Note}

Springer Nature remains neutral with regard to jurisdictional claims in published maps and institutional affiliations.
Received: 24 August 2017 Accepted: 14 January 2018

Published online: 23 January 2018

\section{References}

1. Black DL. Mechanisms of alternative pre-messenger RNA splicing. Annu Rev Biochem. 2003;72:291-336

2. Chen M, Manley JL. Mechanisms of alternative splicing regulation: insights from molecular and genomics approaches. Nat Rev Mol Cell Biol. 2009; 10(11):741-54.

3. Keren H, Lev-Maor G, Ast G. Alternative splicing and evolution: diversification, exon definition and function. Nat Rev Genet. 2010;11(5):345-55.

4. Wang ET, Sandberg R, Luo SJ, Khrebtukova I, Zhang L, Mayr C, Kingsmore SF, Schroth GP, Burge CB. Alternative isoform regulation in human tissue transcriptomes. Nature. 2008;456(7221):470-6.

5. Marquez Y, Brown JW, Simpson C, Barta A, Kalyna M. Transcriptome survey reveals increased complexity of the alternative splicing landscape in Arabidopsis. Genome Res. 2012;22(6):1184-95.

6. Lu XD, Chen DJ, Shu DF, Zhang Z, Wang WX, Klukas C, Chen LL, Fan YL, Chen $M$, Zhang CY. The differential transcription network between embryo and endosperm in the early developing maize seed. Plant Physiol. 2013; 162(1):440-55

7. Li Q, Xiao G, Zhu YX. Single-nucleotide resolution mapping of the Gossypium raimondii transcriptome reveals a new mechanism for alternative splicing of introns. Mol Plant. 2014;7(5):829-40.

8. Wahl MC, Will CL, Luhrmann R. The spliceosome: design principles of a dynamic RNP machine. Cell. 2009:136(4):701-18.

9. Will $\mathrm{CL}$, Luhrmann R. Spliceosome structure and function. CSH Perspect Biol. 2011;3(7): a003707.

10. Bessonov S, Anokhina M, Will CL, Urlaub H, Luhrmann R. Isolation of an active step I spliceosome and composition of its RNP core. Nature. 2008; 452(7189):846-50.

11. Erkelenz S, Mueller WF, Evans MS, Busch A, Schoneweis K, Hertel KJ, Schaal $H$. Position-dependent splicing activation and repression by SR and hnRNP proteins rely on common mechanisms. RNA. 2013;19(1):96-102.

12. Reddy AS. Alternative splicing of pre-messenger RNAs in plants in the genomic era. Annu Rev Plant Biol. 2007;58:267-94.

13. Reddy AS, Shad Ali G. Plant serine/arginine-rich proteins: roles in precursor messenger RNA splicing, plant development, and stress responses. Wires RNA. 2011:2(6):875-89.

14. Luco RF, Allo M, Schor IE, Kornblihtt AR, Misteli T. Epigenetics in alternative pre-mRNA splicing. Cell. 2011;144(1):16-26.

15. ASN R, Marquez Y, Kalyna M, Barta A. Complexity of the alternative splicing landscape in plants. Plant Cell. 2013;25(10):3657-83.

16. Filichkin SA, Priest HD, Givan SA, Shen R, Bryant DW, Fox SE, Wong WK, Mockler TC. Genome-wide mapping of alternative splicing in Arabidopsis Thaliana Genome Res. 2010:20(1):45-58.

17. Ge H, Manley JL. A protein factor, ASF, controls cell-specific alternative splicing of SV40 early pre-mRNA in vitro. Cell. 1990;62(1):25-34.

18. Munoz MJ, Santangelo MSP, Paronetto MP, de la Mata M, Pelisch F, Boireau S, Glover-Cutter K, Ben-Dov C, Blaustein M, Lozano JJ, et al. DNA damage regulates alternative splicing through inhibition of RNA polymerase II elongation. Cell. 2009;137(4):708-20.

19. Sugliani M, Brambilla V, Clerkx EJ, Koornneef M, Soppe WJ. The conserved splicing factor SUA controls alternative splicing of the developmental regulator ABI3 in Arabidopsis. Plant Cell. 2010;22(6):1936-46.

20. Hillier LW, Reinke V, Green P, Hirst M, Marra MA, Waterston RH. Massively parallel sequencing of the polyadenylated transcriptome of $C$. elegans. Genome Res. 2009;19(4):657-66.

21. Lu T, Lu G, Fan D, Zhu C, Li W, Zhao Q, Feng Q, Zhao Y, Guo Y, Li W, et al Function annotation of the rice transcriptome at single-nucleotide resolution by RNA-seq. Genome Res. 2010;20(9):1238-49.

22. Shen Y, Zhou Z, Wang Z, Li W, Fang C, Wu M, Ma Y, Liu T, Kong LA, Peng DL, et al. Global dissection of alternative splicing in paleopolyploid soybean. Plant Cell. 2014;26(3):996-1008

23. Ding F, Cui $P$, Wang Z, Zhang S, Ali S, Xiong L. Genome-wide analysis of alternative splicing of pre-mRNA under salt stress in Arabidopsis. BMC Genomics. 2014;15:431.

24. Chang CY, Lin WD, Tu SL. Genome-wide analysis of heat-sensitive alternative splicing in Physcomitrella patens. Plant Physiol. 2014;165(2):826-40. 
25. Barta A, Kalyna M, Reddy AS. Implementing a rational and consistent nomenclature for serine/arginine-rich protein splicing factors (SR proteins) in plants. Plant Cell. 2010;22(9):2926-9.

26. Zhang F, Zhu G, Du L, Shang X, Cheng C, Yang B, Hu Y, Cai C, Guo W. Genetic regulation of salt stress tolerance revealed by RNA-Seq in cotton diploid wild species, Gossypium davidsonii. Sci Rep. 2016;6:20582.

27. Fagerberg L, Oksvold P, Skogs M, Algenas C, Lundberg E, Ponten F, Sivertsson A, Odeberg J, Klevebring D, Kampf C, et al. Contribution of antibody-based protein profiling to the human Chromosome-centric Proteome Project (C-HPP). J Proteome Res. 2013;12(6):2439-48.

28. Hebenstreit D, Fang M, Gu M, Charoensawan V, van Oudenaarden A, Teichmann SA. RNA sequencing reveals two major classes of gene expression levels in metazoan cells. Mol Syst Biol. 2011;7:497.

29. Vogel C, Marcotte EM. Insights into the regulation of protein abundance from proteomic and transcriptomic analyses. Nat Rev Genet. 2012;13(4):227-32.

30. Thatcher SR, Zhou W, Leonard A, Wang BB, Beatty M, Zastrow-Hayes G, Zhao X, Baumgarten A, Li B. Genome-wide analysis of alternative splicing in Zea mays: landscape and genetic regulation. Plant Cell. 2014;26(9):3472-87.

31. Vitulo N, Forcato C, Carpinelli EC, Telatin A, Campagna D, D'Angelo M, Zimbello R, Corso M, Vannozzi A, Bonghi C, et al. A deep survey of alternative splicing in grape reveals changes in the splicing machinery related to tissue, stress condition and genotype. BMC Plant Biol. 2014;14:99.

32. Li WF, Lin WD, Ray P, Lan P, Schmidt W. Genome-wide detection of condition-sensitive alternative splicing in Arabidopsis roots. Plant Physiol. 2013;162(3):1750-63

33. Zhang GJ, Guo GW, Hu XD, Zhang Y, Li QY, Li RQ, Zhuang RH, Lu ZK, He ZQ, Fang $X D$, et al. Deep RNA sequencing at single base-pair resolution reveals high complexity of the rice transcriptome. Genome Res. 2010;20(5):646-54.

34. Campbell MA, Haas BJ, Hamilton JP, Mount SM, Buell CR. Comprehensive analysis of alternative splicing in rice and comparative analyses with Arabidopsis. BMC Genomics. 2006;7:327.

35. Akerman M, Mandel-Gutfreund $Y$. Alternative splicing regulation at tandem 3' splice sites. Nucleic Acids Res. 2006;34(1):23-31.

36. Himuro Y, Ishiyama K, Mori F, Gondo T, Takahashi F, Shinozaki K, Kobayashi M, Akashi R. Arabidopsis galactinol synthase AtGolS2 improves drought tolerance in the monocot model Brachypodium distachyon. J Plant Physiol. 2014;171(13):1127-31.

37. Verbruggen $\mathrm{N}$, Hermans C. Proline accumulation in plants: a review. Amino Acids. 2008;35(4):753-9.

38. Hamamoto S, Marui J, Matsuoka K, Higashi K, Igarashi K, Nakagawa T, Kuroda T, Mori Y, Murata Y, Nakanishi Y, et al. Characterization of a tobacco TPK-type K+ channel as a novel tonoplast $\mathrm{K}+$ channel using yeast tonoplasts. J Biol Chem. 2008;283(4):1911-20.

39. Zhao YY, Yan F, Hu LP, Zhou XT, Zou ZR, Cui LR. Effects of exogenous 5aminolevulinic acid on photosynthesis, stomatal conductance, transpiration rate, and PIP gene expression of tomato seedlings subject to salinity stress. Genet Mol Res. 2015;14(2):6401-12.

40. Wei Y, Shi H, Xia Z, Tie W, Ding Z, Yan Y, Wang W, Hu W, Li K. Genomewide identification and expression analysis of the WRKY gene family in cassava. Front Plant Sci. 2016;7:25.

41. Cui MH, Yoo KS, Hyoung S, Nguyen HTK, Kim YY, Kim HJ, Ok SH, Yoo SD, Shin JS. An Arabidopsis R2R3-MYB transcription factor, AtMYB20, negatively regulates type $2 C$ serine/threonine protein phosphatases to enhance salt tolerance. FEBS Lett. 2013;587(12):1773-8.

42. Babitha KC, Vemanna RS, Nataraja KN, Udayakumar M. Overexpression of EcbHLH57 transcription factor from Eleusine coracana L. in tobacco confers tolerance to salt, oxidative and drought stress. PLoS One. 2015;10(9):e0137098.

43. Palusa SG, Ali GS, Reddy AS. Alternative splicing of pre-mRNAs of Arabidopsis serine/arginine-rich proteins: regulation by hormones and stresses. Plant J. 2007:49(6):1091-107.

44. Graveley BR, Hertel KJ, Maniatis T. Correspondence - SR proteins are 'locators' of the RNA splicing machinery. Curr Biol. 1999;9(1):R6-7.

45. Kakumanu A, Ambavaram MM, Klumas C, Krishnan A, Batlang U, Myers E, Grene $R$, Pereira A. Effects of drought on gene expression in maize reproductive and leaf meristem tissue revealed by RNA-Seq. Plant Physiol. 2012;160(2):846-67.

46. Singh R, Pandey N, Kumar A, Shirke PA. Physiological performance and differential expression profiling of genes associated with drought tolerance in root tissue of four contrasting varieties of two Gossypium species. Protoplasma. 2016;253(1):163-74.
47. Berget SM, Moore C, Sharp PA. Spliced segments at the $5^{\prime}$ terminus of adenovirus 2 late mRNA. Proc Natl Acad Sci USA. 1977;74(8):3171-5.

48. Mastrangelo AM, Marone D, Laido G, De Leonardis AM, De Vita P. Alternative splicing: enhancing ability to cope with stress via transcriptome plasticity. Plant Sci. 2012;185-186:40-9.

49. Gan X, Stegle O, Behr J, Steffen JG, Drewe P, Hildebrand KL, Lyngsoe R, Schultheiss SJ, Osborne EJ, Sreedharan VT, et al. Multiple reference genomes and transcriptomes for Arabidopsis thaliana. Nature. 2011;477(7365):419-23.

50. Thatcher SR, Danilevskaya ON, Meng X, Beatty M, Zastrow-Hayes G, Harris C, Van Allen B, Habben J, Li B. Genome-wide analysis of alternative splicing during development and drought stress in maize. Plant Physiol. 2016;170(1):586-99.

51. Chung JS, Zhu JK, Bressan RA, Hasegawa PM, Shi H. Reactive oxygen species mediate Na+-induced SOS1 mRNA stability in Arabidopsis. Plant J. 2008; 53(3):554-65.

52. Shi H, Ishitani M, Kim C, Zhu JK. The Arabidopsis thaliana salt tolerance gene SOS1 encodes a putative $\mathrm{Na}+/ \mathrm{H}+$ antiporter. Proc Natl Acad Sci USA. 2000;97(12):6896-901.

53. Saltzman $A L$, Pan $Q$, Blencowe BJ. Regulation of alternative splicing by the core spliceosomal machinery. Genes Dev. 2011;25(4):373-84.

54. Thomas J, Palusa SG, Prasad KV, Ali GS, Surabhi GK, Ben-Hur A, Abdel-Ghany SE, Reddy AS. Identification of an intronic splicing regulatory element involved in auto-regulation of alternative splicing of SCL33 pre-mRNA. Plant J. 2012;72(6):935-46.

55. Marquez Y, Hopfler M, Ayatollahi Z, Barta A, Kalyna M. Unmasking alternative splicing inside protein-coding exons defines exitrons and their role in proteome plasticity. Genome Res. 2015;25(7):995-1007.

56. Yu H, Tian C, Yu Y, Jiao Y. Transcriptome survey of the contribution of alternative splicing to proteome diversity in Arabidopsis thaliana. Mol Plant. 2016;9(5):749-52

57. Carvalho RF, Feijao CV, Duque P. On the physiological significance of alternative splicing events in higher plants. Protoplasma. 2013;250(3):639-50.

58. Duque P. A role for SR proteins in plant stress responses. Plant Signal Behav. 2011:6(1):49-54

59. Isshiki M, Tsumoto A, Shimamoto K. The serine/arginine-rich protein family in rice plays important roles in constitutive and alternative splicing of premRNA. Plant Cell. 2006;18(1):146-58.

60. Feng J, Li J, Gao Z, Lu Y, Yu J, Zheng Q, Yan S, Zhang W, He H, Ma L, et al. SKIP confers osmotic tolerance during salt stress by controlling alternative gene splicing in Arabidopsis. Mol Plant. 2015;8(7):1038-52.

61. Zhu JK. Cell signaling under salt, water and cold stresses. Curr Opin Plant Biol. 2001;4(5):401-6.

62. Schmidt R, Mieulet D, Hubberten HM, Obata T, Hoefgen R, Fernie AR, Fisahn J, San Segundo B, Guiderdoni E, Schippers JH, et al. Salt-responsive ERF1 regulates reactive oxygen species-dependent signaling during the initial response to salt stress in rice. Plant Cell. 2013;25(6):2115-31.

63. Chen S, Jiang J, Li H, Liu G. The salt-responsive transcriptome of Populus simonii x Populus nigra via DGE. Gene. 2012;504(2):203-12.

64. Cui P, Zhang S, Ding F, Ali S, Xiong L. Dynamic regulation of genome-wide pre-mRNA splicing and stress tolerance by the Sm-like protein LSm5 in Arabidopsis. Genome Biol. 2014;15(1):R1.

65. Braunschweig U, Gueroussov S, Plocik AM, Graveley BR, Blencowe BJ. Dynamic integration of splicing within gene regulatory pathways. Cell. 2013; 152(6):1252-69.

66. Syed NH, Kalyna M, Marquez Y, Barta A, Brown JWS. Alternative splicing in plants - coming of age. Trends Plant Sci. 2012;17(10):616-23.

67. Chang YF, Imam JS, Wilkinson MF. The nonsense-mediated decay RNA surveillance pathway. Annu Rev Biochem. 2007;76:51-74.

68. Chen L, Bush SJ, Tovar-Corona JM, Castillo-Morales A, Urrutia AO. Correcting for differential transcript coverage reveals a strong relationship between alternative splicing and organism complexity. Mol Biol Evol. 2014;31(6):1402-13.

69. Flowers TJ. Improving crop salt tolerance. J Exp Bot. 2004;55(396):307-19.

70. Cramer GR, Nowak RS. Supplemental manganese improves the relative growth, net assimilation and photosynthetic rates of salt-stressed barley. Physiol Plantarum. 1992;84(4):600-5.

71. Zhang $T$, Jiang J. Extraction of total RNA in cotton tissues with CTAB-acidic phenolic method. Cotton Sci. 2003;15:166-7.

72. Patel RK, Jain M. NGS QC toolkit: a toolkit for quality control of next generation sequencing data. PLoS One. 2012;7(2):e30619.

73. Trapnell C, Pachter L, Salzberg SL. TopHat: discovering splice junctions with RNA-Seq. Bioinformatics. 2009;25(9):1105-11. 
74. Trapnell C, Roberts A, Goff L, Pertea G, Kim D, Kelley DR, Pimentel H, Salzberg SL, Rinn JL, Pachter L. Differential gene and transcript expression analysis of RNA-seq experiments with TopHat and Cufflinks. Nat Protoc. 2012:7(3):562-78.

75. Sammeth M. Complete alternative splicing events are bubbles in splicing graphs. J Comput Biol. 2009;16(8):1117-40.

76. Du Z, Zhou X, Ling Y, Zhang Z, Su Z. agriGO: a GO analysis toolkit for the agricultural community. Nucleic Acids Res. 2010;38:W64-70.

77. Xu YH, Wang JW, Wang S, Wang JY, Chen XY. Characterization of GaWRKYY, a cotton transcription factor that regulates the sesquiterpene synthase gene (+)-delta-cadinene synthase-A. Plant Physiol. 2004;135:507-15.

78. Livak KJ, Schmittgen TD. Analysis of relative gene expression data using real-time quantitative PCR and the 2(T)(-Delta Delta C) method. Methods. 2001;25(4):402-8.

Submit your next manuscript to BioMed Central and we will help you at every step:

- We accept pre-submission inquiries

- Our selector tool helps you to find the most relevant journal

- We provide round the clock customer support

- Convenient online submission

- Thorough peer review

- Inclusion in PubMed and all major indexing services

- Maximum visibility for your research

Submit your manuscript at www.biomedcentral.com/submit
Biomed Central 